\title{
AVALIAÇÃO DA FUNÇÃO MOTORA EM CRIANÇAS COM DISTROFIA MUSCULAR CONGÊNITA COM DEFICIÊNCIA DA MEROSINA
}

\author{
Fernanda M. Rocco', Fernanda H. Gianini Luz', \\ Alexsander Junquera Rossato ${ }^{2}$, Antônio Carlos Fernandes ${ }^{3}$, \\ Acary S.B. Oliveira ${ }^{4}$, Javier Toledano Beteta ${ }^{5}$, Edmar Zanoteli ${ }^{5}$
}

\begin{abstract}
RESUMO - A distrofia muscular congênita (DMC) compõe um grupo de miopatias caracterizadas por hipotonia e fraqueza muscular notadas até o primeiro ano de vida. Em torno de $40 \%$ a $50 \%$ dos casos são decorrentes de deficiência primária da proteína merosina (DM), os quais apresentam um fenótipo mais homogêneo, com grave comprometimento motor e respiratório. Foram avaliadas neste estudo onze crianças com diagnóstico clínico e histológico de DMC-DM, com idade de 3 a 15 anos, através de exame de força muscular ("Medical Research Council"), análise goniométrica, avaliação das habilidades motoras e das atividades de vida diária (AVDs) (indicador de Barthel), com o objetivo de caracterizar as principais limitações funcionais motoras. Os grupos musculares mais comprometidos foram os flexores cervicais, paravertebrais e proximais dos membros. Os grupos musculares dos membros superiores estavam tão comprometidos quanto os dos membros inferiores, enquanto que os extensores encontravam-se mais comprometidos que os flexores. Todas as crianças apresentavam importantes retrações musculares nos quadris, joelhos e cotovelos. Outras deformidades freqüentes foram escoliose e pés eqüino-varo. Nenhuma criança possuía a habilidade motora necessária para engatinhar, ficar de pé ou andar; e todas foram classificadas como dependentes ou semidependentes para a maioria das AVDs estudadas. Nossos achados confirmam o envolvimento difuso e intenso da musculatura esquelética na DMC-DM, acarretando graves limitações funcionais motoras e deformidades músculo-esqueléticas.
\end{abstract}

PALAVRAS-CHAVE: distrofia muscular congênita, merosina, miopatias, retrações musculares, reabilitação.

\begin{abstract}
Motor function evaluation in merosin-deficient congenital muscular dystrophy children
ABSTRACT - Congenital muscular dystrophy (CMD) is a heterogeneous group of disorders characterized by early onset of hypotonia and weakness. Almost $50 \%$ of the cases are caused by primary deficiency of a protein named merosin (MD), and present a homogenous phenotype with a severe motor and respiratory involvement. Eleven children with clinical and histological diagnosis of CMD-MD, aged of 3 to 15 years, were studied using the manual muscle testing (Medical Research Council), goniometric analysis, motor ability and day life activities (Barthel index) scales, with the objective to characterize the main motor function limitations. The muscular groups most affected were cervical flexors, paravertebral and proximal portions of limbs. The muscular groups of upper limbs were as affected as the lower limbs, and the extensors were more affected than the flexors groups. All children had severe muscular retractions on the hip, knee and elbow. Other frequent deformities were scoliosis and equinus-varum feet. No children presented the motor ability to walk, stand up and crawl; and all of them were classified as dependents or semi-dependents in the day life activities scale. Our findings confirm the severe and diffuse involvement of skeletal muscle in CMD-MD patients, producing serious motor limitations and deformities.
\end{abstract}

KEY WORDS: congenital muscular dystrophy, merosin, myopathy, muscle retractions, rehabilitation.

A distrofia muscular congênita (DMC) compõe um grupo de miopatias caracterizadas por comprometimento muscular notado já ao nascimento, ou no primeiro ano de vida. $O$ tecido muscular apresen- ta-se distrófico e sem substrato histopatológico específico ${ }^{1,2}$. As principais características clínicas incluem hipotonia, atrofia e fraqueza musculares estacionárias ou com mínima progressão, associadas com

Clínica de Doenças Neuromusculares da Associação de Assistência à Criança Deficiente (AACD), São Paulo SP, Brasil: ${ }^{1}$ Médica Residente, ${ }^{2}$ Ortopedista, ${ }^{3}$ Diretor Clínico, ${ }^{5}$ Neurologista Disciplina de Neurologia da Universidade Federal de São Paulo, Escola Paulista de Medicina, São Paulo SP, Brasil (EPM); ${ }^{4}$ Neurologista, Chefe do Setor de Doenças Neuromusculares da EPM.

Recebido 12 Julho 2004, recebido na forma final 17 Setembro 2004. Aceito 9 Novembro 2004.

Dra. Fernanda M. Rocco - Divisão Médica, AACD - Avenida Prof. Ascendino Reis 724 - 04027-000 São Paulo SP - Brasil. E-mail: roccofilho@sti.com.br 
deformidades músculo-esqueléticas diversas ${ }^{1,2}$. A fraqueza muscular predomina nas porções proximais dos membros. Os músculos paravertebrais, cervicais, mastigatórios e faciais são também acometidos $^{1,2}$. A eletroneuromiografia revela na maioria dos casos um padrão tipicamente miopático ${ }^{1,2}$. $O$ prognóstico depende do grau do comprometimento da musculatura respiratória e do desenvolvimento de deformidades da coluna vertebral ${ }^{1-3}$. Vários genes estão envolvidos nesta síndrome ${ }^{2-4}$. Quase a metade dos casos é causada por mutações no gene da cadeia $\alpha 2$ da laminina-2, chamada de merosina, uma proteína da matriz extracelular que se relaciona com as proteínas intracitoplasmáticas, como a distrofina, através de interações com o complexo distroglicano na membrana da fibra muscular $^{2,3}$. Outras formas mais raras de DMC são causadas pela deficiência genética de diversas proteínas, tais como o colágeno VI [genes COL6A1 e COL 6A2 (21q22) e COL6A3 (2q37)] (DMC forma de Ullrich); integrina a7 (gene ITGA7, 12q); selenoproteínaN1 (gene SPT1, 1p35) (DMC forma com espinha rígida); fukutina-relacionada (gene FKRP, 19q1) (DM C1C); proteína "acetilglucosaminiltransferase-like" (gene LARGE, 22q) (DMC1D); fukutina (gene Fukutin, 9q31) (doença de Fukuyama); O-manose $\beta-1,2-\mathrm{N}$-acetilglucosamiltransferase (gene POMGnT1, 1p33) (doença músculo-olho-cérebro) e O-mannosyltransferase 1 (gene POMT1, 9q34) (doença de Walker Warburg $^{3,4}$. Existe ainda um grupo heterogêneo de pacientes com DMC, cujo defeito genético, ou a deficiência protéica, ainda não foram identificados. Estes pacientes são incluídos no subgrupo chamado de DMC com merosina presente ${ }^{2,3}$.

Os pacientes com deficiência da merosina (DMCDM), em torno de $40-50 \%$ dos casos, apresentam um fenótipo mais grave ${ }^{5-9}$. A maioria não chega a deambular e o comprometimento respiratório é mais intenso. Outro achado característico nesses pacientes é o comprometimento da substância branca do sistema nervoso central (SNC), embora o nível cognitivo seja normal na maioria das crianças ${ }^{3,7,9,10}$. Até o presente momento não há tratamento defini- tivo para estas crianças. No entanto, o tratamento fisioterápico quando iniciado precocemente tende a promover melhora na qualidade de vida e da sobrevida destas crianças ${ }^{11,12}$. Uma avaliação funcional detalhada, incluindo-se exame de força muscular, detecção de deformidades músculo-esqueléticas, avaliação da capacidade funcional e provas de função pulmonar, são fundamentais no sentido de promover um programa amplo de reabilitação ${ }^{13}$.

O objetivo deste estudo foi o de determinar as principais incapacidades funcionais motoras em crianças com DMC-DM, especialmente quanto aos grupos musculares mais afetados, as habilidades motoras mais comprometidas, as principais deformidades músculo-esqueléticas e as limitações nas atividades de vida diária.

\section{MÉTODO}

Foram incluídos neste estudo onze crianças com diagnóstico clínico e histológico de DMC com deficiência da proteína merosina. Destas, três eram do sexo feminino e oito do masculino, com idade variando entre 3 e 15 anos, com média de 8 anos (Tabela 1). A época do início das manifestações ocorreu antes dos 6 meses de vida em todos os casos. Todas as crianças foram avaliadas, em pelo menos uma oportunidade, por dois examinadores diferentes.

Todas as crianças tiveram o diagnóstico histológico de DMC-DM através de biópsia muscular com estudo imunohistoquímico. Os principais achados histológicos observados foram variabilidade no tamanho das fibras, fibras musculares atrofiadas com aspecto arredondado, aumento do tecido conjuntivo endomisial e perimisial, raras centralizações nucleares, alterações da arquitetura interna das fibras e raras fibras em necrose e macrofagia. Não foram observadas alterações estruturais específicas tais como corpos nemalínicos, "cores" centrais, inclusões e "ragged-red fibers". A análise imunohistoquímica através de imunofluorescência indireta revelou ausência de marcação do anticorpo contra a proteína merosina (porção $300 \mathrm{kDa}$ da cadeia $\alpha 2$ da laminina, anti-mouse, clone Mer 3/22B2, Novocastra), na diluição de 1:100. A marcação para anticorpos contra outras proteínas, tais como distrofina (Novocastra, porção C-terminal, anti-mouse, diluição 1:20), adalina (Novocastra, anti-mouse, diluição 1:100) e disferlina (Novocastra, anti-mouse, diluição 1:20) encon-

Tabela 1. Dados gerais em onze crianças portadoras de distrofia muscular congênita com deficiência da merosina.

\begin{tabular}{lccccccccccc}
\hline Pacientes & 1 & 2 & 3 & 4 & 5 & 6 & 7 & 8 & 9 & 10 & 11 \\
\hline Idade (anos) & 9 & 8 & 12 & 13 & 3 & 5 & 11 & 6 & 5 & 8 & 15 \\
Sexo & $\mathrm{M}$ & $\mathrm{F}$ & $\mathrm{M}$ & $\mathrm{M}$ & $\mathrm{M}$ & $\mathrm{M}$ & $\mathrm{F}$ & $\mathrm{M}$ & $\mathrm{F}$ & $\mathrm{M}$ & $\mathrm{M}$ \\
Terapias na AACD & não & não & $\mathrm{sim}$ & não & não & sim & não & sim & não & sim & não \\
\hline M masculino: F, fminino & & & & & & & & & & &
\end{tabular}




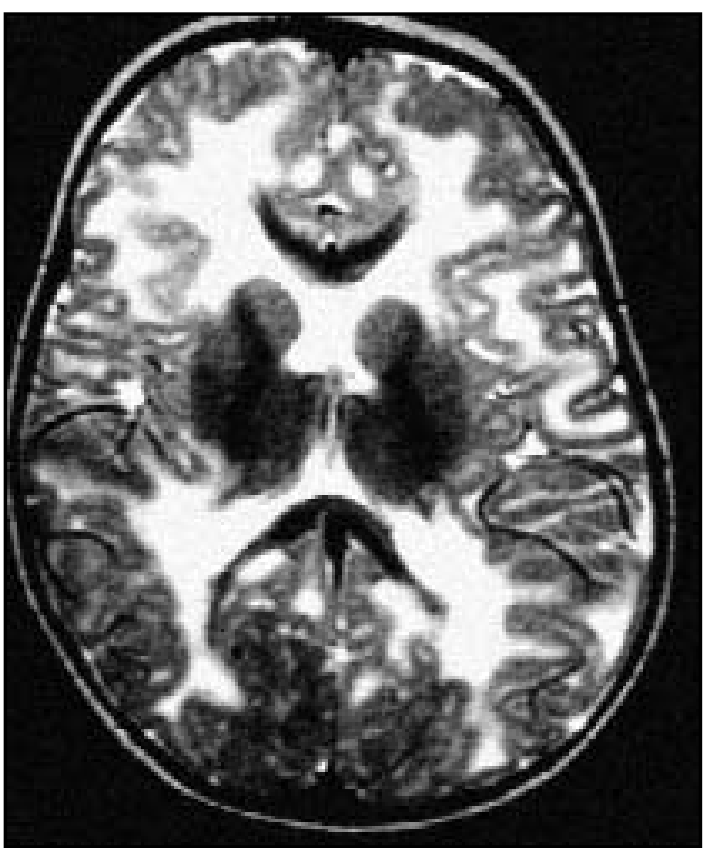

Fig 1. Imagem coronal de RM de crânio (T2-WI) revelando a presença de hipersinal na substância branca periventricular nas regiões frontais, parietais e occipitais (Caso 3).

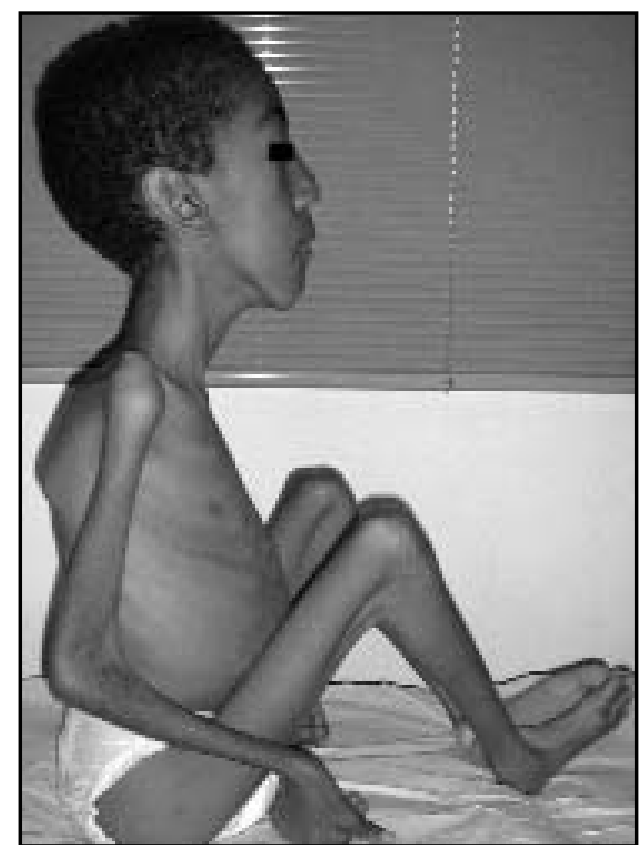

Fig 2. Criança com distrofia muscular congênita com deficiência da merosina (Caso 3). Notar a presença de retrações musculares nas articulações dos cotovelos, quadris e joelhos; e a atrofia muscular cervical e proximal no membro superior. trou-se normal em todos os casos. Foi utilizado como anticorpo secundário o FITC anti-mouse.

Informações de exames subsidiários (raio-X da coluna vertebral e prova de função respiratória), realizados previamente, foram obtidas dos prontuários. Todos os pacientes tinham ressonância magnética (RM) e/ou tomografia computadorizada (TC) de crânio, e os principais achados incluíam anormalidades difusas da substância branca, afetando o centro semi-oval, especialmente, nas topografias periventriculares e cerebelares (Fig 1). Não foram observadas atrofia cerebral ou cerebelar, ou defeitos de migração neuronal.

Este estudo foi avaliado e aprovado pelo Conselho de Ética Médica do Hospital da AACD.

Exame da força muscular - Os grupos musculares flexores e extensores de pescoço, tronco, ombros, cotovelos, punhos, quadris, joelhos e tornozelos; abdutores e adutores de ombros e quadris; eversores e inversores dos pés foram avaliados através da escala "Medical Research Council" (MRC) ${ }^{14,15}$.

Avaliação goniométrica da amplitude dos movimentos das articulações - Foi avaliada de forma passiva, utilizando um goniômetro manual, a amplitude dos movimentos das articulações dos ombros (adução e abdução), cotovelos (flexão e extensão), punhos (flexão e extensão), quadris (flexão, extensão, abdução e adução), joelhos (flexão e extensão), e tornozelos (flexão, extensão, eversão e inversão), bem como os movimentos cervicais (flexão, extensão e rotação lateral), seguindo critérios e valores normais propostos pela "American Academy of Orthopedic Surgeons"16,17. As amplitudes consideradas normais estão apresentadas na primeira coluna da Tabela 3. Também foi avaliado o grau de retração muscular dos flexores dos quadris pelo teste de Thomas ${ }^{17}$. Em todos os movimentos foi considerado como normal uma variação de até $5 \%$. Foram anotados os valores angulares apenas daqueles movimentos articulares que se encontravam reduzidos (menor que o limite superior de normalidade).

Avaliação da capacidade funcional motora - A análise da capacidade funcional motora foi realizada aplicandose uma escala utilizada por Russman et al. em pacientes com amiotrofia espinhal, que avalia de forma qualitativa e descritiva as habilidades motoras para membros inferiores (sentar, engatinhar, ficar de pé, andar, subir e descer escadas), membros superiores (elevação dos braços, flexão de antebraços e flexão e extensão dos punhos) e cervicais (capacidade para elevação e/ou para o sustento cervical) ${ }^{18}$.

Avaliação das atividades da vida diária (AVDs) - Para a avaliação das AVDs foi utilizado o indicador de Barthel (IB), que analisa a capacidade do paciente para realizar 10 itens (Tabela 5), de forma independente, com grande ou pequena ajuda ou totalmente dependente ${ }^{19}$. 
Tabela 2. Grau de força muscular de acordo com a escala MRC em onze crianças portadoras de distrofia muscular congênita com deficiência da merosina.

\begin{tabular}{|c|c|c|c|c|c|c|c|c|c|c|c|c|}
\hline $\begin{array}{l}\text { Grupos } \\
\text { musculares }\end{array}$ & 1 & 2 & 3 & 4 & 5 & 6 & 7 & 8 & 9 & 10 & 11 & Média \\
\hline Flex Pescoço & 2 & 2 & 2 & 2 & 2 & 2 & 2 & 2 & 2 & 2 & 2 & 2 \\
\hline Ext Pescoço & 2 & 2 & 2 & 2 & 2 & 2 & 2 & 2 & 2 & 2 & 2 & 2 \\
\hline Flex Tronco & 2 & 2 & 2 & 2 & 2 & 2 & 2 & 2 & 2 & 2 & 2 & 2 \\
\hline Ext Tronco & 2 & 2 & 2 & 2 & 2 & 2 & 2 & 2 & 2 & 2 & 2 & 2 \\
\hline Abd Ombro & $\begin{array}{l}D=1 \\
E=3\end{array}$ & $\mathrm{D}=\mathrm{E}=2$ & $\mathrm{D}=\mathrm{E}=2$ & $\mathrm{D}=\mathrm{E}=2$ & $\mathrm{D}=\mathrm{E}=2$ & $\mathrm{D}=\mathrm{E}=3$ & $\mathrm{D}=\mathrm{E}=3$ & $\mathrm{D}=\mathrm{E}=2$ & $\mathrm{D}=\mathrm{E}=2$ & $\mathrm{D}=\mathrm{E}=2$ & $\mathrm{D}=\mathrm{E}=2$ & 2,18 \\
\hline Ad Ombro & $\begin{array}{l}D=1 \\
E=3\end{array}$ & $\mathrm{D}=\mathrm{E}=2$ & $\mathrm{D}=\mathrm{E}=2$ & $\mathrm{D}=\mathrm{E}=2$ & $\mathrm{D}=\mathrm{E}=2$ & $\mathrm{D}=\mathrm{E}=3$ & $D=E=3$ & $\mathrm{D}=\mathrm{E}=2$ & $\mathrm{D}=\mathrm{E}=2$ & $\mathrm{D}=\mathrm{E}=2$ & $\mathrm{D}=\mathrm{E}=2$ & 2,18 \\
\hline Flex Ombro & $\begin{array}{l}D=1 \\
E=3\end{array}$ & $\mathrm{D}=\mathrm{E}=2$ & $\mathrm{D}=\mathrm{E}=2$ & $\mathrm{D}=\mathrm{E}=2$ & $\mathrm{D}=\mathrm{E}=2$ & $\mathrm{D}=\mathrm{E}=3$ & $\mathrm{D}=\mathrm{E}=3$ & $\mathrm{D}=\mathrm{E}=2$ & $\mathrm{D}=\mathrm{E}=2$ & $\mathrm{D}=\mathrm{E}=2$ & $\mathrm{D}=\mathrm{E}=2$ & 2,18 \\
\hline Ext Ombro & $\begin{array}{l}D=1 \\
E=3\end{array}$ & $\mathrm{D}=\mathrm{E}=2$ & $\mathrm{D}=\mathrm{E}=2$ & $\mathrm{D}=\mathrm{E}=2$ & $\mathrm{D}=\mathrm{E}=2$ & $\mathrm{D}=\mathrm{E}=3$ & $\mathrm{D}=\mathrm{E}=4$ & $\mathrm{D}=\mathrm{E}=2$ & $\mathrm{D}=\mathrm{E}=2$ & $\mathrm{D}=\mathrm{E}=2$ & $\mathrm{D}=\mathrm{E}=2$ & 2,27 \\
\hline Flex Cotovelo & $\mathrm{D}=\mathrm{E}=3$ & $\mathrm{D}=\mathrm{E}=3$ & $\mathrm{D}=\mathrm{E}=4$ & $\mathrm{D}=\mathrm{E}=4$ & $\mathrm{D}=\mathrm{E}=3$ & $\mathrm{D}=\mathrm{E}=3$ & $\mathrm{D}=\mathrm{E}=4$ & $\mathrm{D}=\mathrm{E}=3$ & $\mathrm{D}=\mathrm{E}=2$ & $\mathrm{D}=\mathrm{E}=3$ & $\mathrm{D}=\mathrm{E}=3$ & 3,18 \\
\hline Ext Cotovelo & $\mathrm{D}=\mathrm{E}=2$ & $\mathrm{D}=\mathrm{E}=3$ & $\mathrm{D}=\mathrm{E}=2$ & $\mathrm{D}=\mathrm{E}=3$ & $\mathrm{D}=\mathrm{E}=3$ & $\mathrm{D}=\mathrm{E}=3$ & $\mathrm{D}=\mathrm{E}=4$ & $\mathrm{D}=\mathrm{E}=2$ & $\mathrm{D}=\mathrm{E}=2$ & $\mathrm{D}=\mathrm{E}=2$ & $\mathrm{D}=\mathrm{E}=3$ & 2,6 \\
\hline Ext Punho & $\mathrm{D}=\mathrm{E}=4$ & $\mathrm{D}=\mathrm{E}=3$ & $\mathrm{D}=\mathrm{E}=4$ & $\mathrm{D}=\mathrm{E}=4$ & $\mathrm{D}=\mathrm{E}=4$ & $\mathrm{D}=\mathrm{E}=3$ & $\mathrm{D}=\mathrm{E}=4$ & $\mathrm{D}=\mathrm{E}=3$ & $\begin{array}{l}D=3 \\
E=2\end{array}$ & $\mathrm{D}=\mathrm{E}=3$ & $\begin{array}{c}\mathrm{D}=\mathrm{E}= \\
\mathrm{NA}\end{array}$ & 3,4 \\
\hline Flex Punho & $\mathrm{D}=\mathrm{E}=4$ & NA & $\mathrm{D}=\mathrm{E}=2$ & $\mathrm{D}=\mathrm{E}=4$ & $\mathrm{D}=\mathrm{E}=4$ & $\mathrm{D}=\mathrm{E}=3$ & $\mathrm{D}=\mathrm{E}=4$ & NA & NA & $\begin{array}{c}D=4 \\
E=N A\end{array}$ & $\mathrm{D}=\mathrm{E}=4$ & 3,6 \\
\hline Flex Quadril & $\mathrm{D}=\mathrm{E}=3$ & $\mathrm{D}=\mathrm{E}=3$ & $\mathrm{D}=\mathrm{E}=4$ & $\mathrm{D}=\mathrm{E}=2$ & $\begin{array}{l}D=2 \\
E=3\end{array}$ & $\mathrm{D}=\mathrm{E}=3$ & $\mathrm{D}=\mathrm{E}=3$ & $\mathrm{D}=\mathrm{E}=3$ & $\mathrm{D}=\mathrm{E}=2$ & $\mathrm{D}=\mathrm{E}=3$ & $\mathrm{D}=\mathrm{E}=2$ & 2,7 \\
\hline Ext Quadril & $\mathrm{D}=\mathrm{E}=2$ & $\mathrm{D}=\mathrm{E}=2$ & $\mathrm{D}=\mathrm{E}=2$ & $\mathrm{D}=\mathrm{E}=2$ & $\mathrm{D}=\mathrm{E}=3$ & $\mathrm{D}=\mathrm{E}=3$ & $\mathrm{D}=\mathrm{E}=2$ & $\mathrm{D}=\mathrm{E}=2$ & $\mathrm{D}=\mathrm{E}=2$ & $\mathrm{D}=\mathrm{E}=3$ & $\mathrm{D}=\mathrm{E}=2$ & 2,27 \\
\hline Abd Quadril & $\mathrm{D}=\mathrm{E}=3$ & $\mathrm{D}=\mathrm{E}=3$ & $\mathrm{D}=\mathrm{E}=3$ & $\mathrm{D}=\mathrm{E}=3$ & $\mathrm{D}=\mathrm{E}=3$ & $\mathrm{D}=\mathrm{E}=3$ & $\mathrm{D}=\mathrm{E}=2$ & $\mathrm{D}=\mathrm{E}=2$ & $\mathrm{D}=\mathrm{E}=2$ & $\mathrm{D}=\mathrm{E}=3$ & $\mathrm{D}=\mathrm{E}=3$ & 2,7 \\
\hline Ad Quadril & $\begin{array}{l}D=3 \\
E=2\end{array}$ & $\mathrm{D}=\mathrm{E}=3$ & $\mathrm{D}=\mathrm{E}=3$ & $\mathrm{D}=\mathrm{E}=3$ & $\mathrm{D}=\mathrm{E}=3$ & $\mathrm{D}=\mathrm{E}=3$ & $\mathrm{D}=\mathrm{E}=4$ & $\mathrm{D}=\mathrm{E}=2$ & $\mathrm{D}=\mathrm{E}=2$ & $\mathrm{D}=\mathrm{E}=3$ & $\mathrm{D}=\mathrm{E}=3$ & 2,8 \\
\hline Flex Joelho & $\mathrm{D}=\mathrm{E}=4$ & $\mathrm{D}=\mathrm{E}=3$ & $\mathrm{D}=\mathrm{E}=4$ & $\mathrm{D}=\mathrm{E}=3$ & $\begin{array}{l}D=2 \\
E=3\end{array}$ & $\mathrm{D}=\mathrm{E}=3$ & $\mathrm{D}=\mathrm{E}=4$ & $\mathrm{D}=\mathrm{E}=3$ & $\mathrm{D}=\mathrm{E}=3$ & $\mathrm{D}=\mathrm{E}=3$ & $\mathrm{D}=\mathrm{E}=3$ & 3,2 \\
\hline Ext Joelho & $\mathrm{D}=\mathrm{E}=3$ & $\mathrm{D}=\mathrm{E}=3$ & $\begin{array}{l}D=2 \\
E=3\end{array}$ & $\mathrm{D}=\mathrm{E}=3$ & $\begin{array}{l}D=2 \\
E=3\end{array}$ & $\mathrm{D}=\mathrm{E}=3$ & $\mathrm{D}=\mathrm{E}=2$ & $\mathrm{D}=\mathrm{E}=2$ & $\mathrm{D}=\mathrm{E}=4$ & $\mathrm{D}=\mathrm{E}=3$ & $\mathrm{D}=\mathrm{E}=3$ & 2,8 \\
\hline FlexTornozelo & NA & NA & NA & NA & $\mathrm{D}=\mathrm{E}=4$ & $\mathrm{D}=\mathrm{E}=3$ & NA & NA & NA & NA & NA & 3,5 \\
\hline Ext Tornozelo & NA & NA & NA & NA & $\mathrm{D}=\mathrm{E}=4$ & $\mathrm{D}=\mathrm{E}=3$ & NA & NA & NA & NA & NA & 3,5 \\
\hline Eversão Pé & NA & NA & NA & NA & $\mathrm{D}=\mathrm{E}=4$ & $\mathrm{D}=\mathrm{E}=2$ & NA & NA & NA & NA & NA & 3 \\
\hline Inversão Pé & NA & NA & NA & NA & $\mathrm{D}=\mathrm{E}=4$ & $\mathrm{D}=\mathrm{E}=3$ & NA & NA & NA & NA & NA & 3,5 \\
\hline
\end{tabular}

D, direito; E, esquerdo; NA, não avaliável devido à deformidade estruturada; Flex, flexão; Ext, extensão; Abd, abdução; Ad, adução.

\section{RESULTADOS}

Exame de força muscular - A média do grau de força muscular, pelo MRC, para todos os grupos musculares analisados foi 2,7. Como demonstrado na Tabela 2, em todos os casos foi observado maior comprometimento dos grupos musculares proximais dos membros com relação aos distais. A média para os grupos musculares proximais (ombros e quadris) foi 2,4 , enquanto que para os grupos musculares distais (cotovelos, punhos, joelhos e tornozelos) foi 3,2 . Os grupos musculares dos membros superiores (MS) estavam tão comprometidos quanto os dos membros inferiores (MI), sendo a força muscular média para MS 2,7 e para MI 2,9. Grupos musculares extensores (média de força muscular $=2,8$ ) encontra- vam-se mais fracos que os flexores (média de força muscular $=3,1$ ) nas articulações estudadas. Quanto à força dos músculos flexores e extensores do pescoço e do tronco encontramos grau 2 em todos os casos.

Nos casos 2, 8, 9 e 10 (este somente à esquerda) não foi possível avaliar a força muscular para flexão do punho, e no caso 11 para extensão do punho, devido a deformidade fixa na articulação. Em todas as outras crianças, encontramos força muscular geralmente de grau 3 a 4. Em nove crianças (casos 1, $2,3,4,7,8,9,10$ e 11), não foi possível determinar o grau da força muscular nas articulações dos tornozelos devido a deformidade fixa. Nos casos 5 e 6, a força para flexão dorsal e flexão plantar variou de grau 3 a 4. 
Tabela 3. Avaliação goniométrica (amplitude dos movimentos articulares e grau das retrações musculares elou deformidades) em onze crianças com distrofia muscular congênita com deficiência da merosina. Na primeira coluna estão apresentados os valores angulares considerados normais da amplitude das articulações.

\begin{tabular}{|c|c|c|c|c|c|c|c|c|c|c|c|}
\hline Articulação & 1 & 2 & 3 & 4 & 5 & 6 & 7 & 8 & 9 & 10 & 11 \\
\hline Flex cervic $\left(60^{\circ}\right)$ & NL & $0^{\circ}$ & $20^{\circ}$ & $24^{\circ}$ & $42^{\circ}$ & NL & NL & $20^{\circ}$ & $0^{\circ}$ & $\mathrm{NL}$ & $0^{\circ}$ \\
\hline Ext cervic $\left(75^{\circ}\right)$ & NL & NL & $\mathrm{NL}$ & $0^{\circ}$ & NL & NL & NL & $\mathrm{NL}$ & NL & $\mathrm{NL}$ & NL \\
\hline Rot cervic $D\left(80^{\circ}\right)$ & $50^{\circ}$ & NL & $30^{\circ}$ & $35^{\circ}$ & $\mathrm{NL}$ & NL & NL & $38^{\circ}$ & $\mathrm{NL}$ & $\mathrm{NL}$ & $10^{\circ}$ \\
\hline Rot cervic E $\left(80^{\circ}\right)$ & $24^{\circ}$ & NL & $16^{\circ}$ & $10^{\circ}$ & NL & NL & NL & $64^{\circ}$ & $34^{\circ}$ & $\mathrm{NL}$ & $30^{\circ}$ \\
\hline \multirow[t]{2}{*}{ Abd ombro $\left(180^{\circ}\right)$} & NL & D $122^{\circ}$ & D $120^{\circ}$ & D $102^{\circ}$ & NL & $\mathrm{D} 78^{\circ}$ & NL & $\mathrm{D} 112^{\circ}$ & NL & NL & NL \\
\hline & & E $130^{\circ}$ & E $150^{\circ}$ & $\mathrm{E} 90^{\circ}$ & & $\mathrm{E} 80^{\circ}$ & & E $98^{\circ}$ & & & \\
\hline \multirow[t]{2}{*}{ Ad ombro $\left(75^{\circ}\right)$} & $\mathrm{NL}$ & NL & NL & NL & NL & NL & NL & NL & $\mathrm{NL}$ & $\mathrm{NL}$ & $\mathrm{D} 38^{\circ}$ \\
\hline & & & & & & & & & & & E $64^{\circ}$ \\
\hline Cotovelo & D $66^{\circ}$ & D $110^{\circ}$ & D $68^{\circ}$ & $\mathrm{D} 85^{\circ}$ & D $15^{\circ}$ & D $30^{\circ}$ & D $38^{\circ}$ & D $62^{\circ}$ & D $20^{\circ}$ & D $45^{\circ}$ & $\mathrm{D} 90^{\circ}$ \\
\hline \multirow[t]{3}{*}{ (ext-flex $/ 0^{\circ} 140^{\circ}$} & $140^{\circ}$ & $140^{\circ}$ & $140^{\circ}$ & $140^{\circ}$ & $140^{\circ}$ & $135^{\circ}$ & $140^{\circ}$ & $140^{\circ}$ & $140^{\circ}$ & $140^{\circ}$ & $124^{\circ}$ \\
\hline & $\mathrm{E} 74^{\circ}$ & E $115^{\circ}$ & $\mathrm{E} 64^{\circ}$ & $\mathrm{E} 84^{\circ}$ & $\mathrm{E} 26^{\circ}$ & $\mathrm{E} 36^{\circ}$ & $\mathrm{E} 36^{\circ}$ & $\mathrm{E} 65^{\circ}$ & $\mathrm{E} 26^{\circ}$ & $\mathrm{E} 30^{\circ}$ & $\mathrm{E} 100^{\circ}$ \\
\hline & $140^{\circ}$ & $140^{\circ}$ & $140^{\circ}$ & $140^{\circ}$ & $140^{\circ}$ & $138^{\circ}$ & $140^{\circ}$ & $140^{\circ}$ & $140^{\circ}$ & $140^{\circ}$ & $120^{\circ}$ \\
\hline \multirow[t]{2}{*}{ Flexo de cotovelo } & D $66^{\circ}$ & D $110^{\circ}$ & D $68^{\circ}$ & $\mathrm{D} 85^{\circ}$ & D $15^{\circ}$ & D $30^{\circ}$ & D $38^{\circ}$ & D $62^{\circ}$ & D $20^{\circ}$ & D $45^{\circ}$ & $\mathrm{D} 90^{\circ}$ \\
\hline & $\mathrm{E} 74^{\circ}$ & E $115^{\circ}$ & $\mathrm{E} 64^{\circ}$ & $\mathrm{E} 84^{\circ}$ & $\mathrm{E} 26^{\circ}$ & $E 36^{\circ}$ & $E 36^{\circ}$ & $\mathrm{E} 65^{\circ}$ & $\mathrm{E} 26^{\circ}$ & $\mathrm{E} 30^{\circ}$ & $\mathrm{E} 100^{\circ}$ \\
\hline Flex do punho & NL & $\mathrm{D}=\mathrm{E} \infty$ & NL & $\mathrm{NL}$ & NL & NL & NL & D $40^{\circ}$ & $\mathrm{D}=\mathrm{E}$ & $\mathrm{E} 34^{\circ}$ & NL \\
\hline$\left(90^{\circ}\right)$ & & $50^{\circ}$ & & & & & & $\mathrm{E}-14^{\circ}$ & $0^{\circ}$ & D NL & \\
\hline Ext do punho & $\mathrm{NL}$ & NL & NL & NL & NL & NL & NL & $\mathrm{NL}$ & NL & $\mathrm{NL}$ & $\mathrm{D}=\mathrm{E}$ \\
\hline$\left(70^{\circ}\right)$ & & & & & & & & & & & $0^{\circ}$ \\
\hline Quadril & D $53^{\circ}$ & $\mathrm{D} 70^{\circ}$ & $\mathrm{D} 90^{\circ}$ & $\mathrm{D} 48^{\circ}$ & D $12^{\circ}$ & D $45^{\circ}$ & D $12^{\circ}$ & D $40^{\circ}$ & $\mathrm{D} 35^{\circ}$ & D $66^{\circ}$ & D $40^{\circ}$ \\
\hline \multirow[t]{3}{*}{$\left(\right.$ ext-flex/ $\left.0^{\circ} 120^{\circ}\right)$} & $125^{\circ}$ & $125^{\circ}$ & $125^{\circ}$ & $118^{\circ}$ & $125^{\circ}$ & $104^{\circ}$ & $110^{\circ}$ & $125^{\circ}$ & $125^{\circ}$ & $125^{\circ}$ & $92^{\circ}$ \\
\hline & $\mathrm{E} 64^{\circ}$ & $\mathrm{E} 47^{\circ}$ & $\mathrm{E} 90^{\circ}$ & $\mathrm{E} 54^{\circ}$ & $\mathrm{E} 20^{\circ}$ & $\mathrm{E} 45^{\circ}$ & $\mathrm{E} 58^{\circ}$ & $\mathrm{E} 42^{\circ}$ & $\mathrm{E} 46^{\circ}$ & $\mathrm{E} 76^{\circ}$ & $\mathrm{E} 48^{\circ}$ \\
\hline & $125^{\circ}$ & $125^{\circ}$ & $125^{\circ}$ & $122^{\circ}$ & $125^{\circ}$ & $125^{\circ}$ & $113^{\circ}$ & $125^{\circ}$ & $125^{\circ}$ & $125^{\circ}$ & $84^{\circ}$ \\
\hline Flexo de quadril & D $53^{\circ}$ & $\mathrm{D} 70^{\circ}$ & $\mathrm{D} 90^{\circ}$ & D $48^{\circ}$ & D $12^{\circ}$ & D $45^{\circ}$ & D $12^{\circ}$ & D $40^{\circ}$ & $\mathrm{D} 35^{\circ}$ & D $66^{\circ}$ & D $40^{\circ}$ \\
\hline (Thomas) & $\mathrm{E} 64^{\circ}$ & $\mathrm{E} 47^{\circ}$ & $\mathrm{E} 90^{\circ}$ & $\mathrm{E} 54^{\circ}$ & $\mathrm{E} 20^{\circ}$ & $\mathrm{E} 45^{\circ}$ & $E 58^{\circ}$ & $\mathrm{E} 58^{\circ}$ & $\mathrm{E} 46^{\circ}$ & E $76^{\circ}$ & $\mathrm{E} 48^{\circ}$ \\
\hline \multirow[t]{2}{*}{ Abd quadril $\left(50^{\circ}\right)$} & NL & NL & NL & NL & NL & $\mathrm{NL}$ & D $12^{\circ}$ & D $40^{\circ}$ & NL & NL & D $14^{\circ}$ \\
\hline & & & & & & & $\mathrm{E} 8^{\circ}$ & $\mathrm{E} 42^{\circ}$ & & & $\mathrm{E} 20^{\circ}$ \\
\hline Ad do quadril & NL & NL & NL & NL & NL & NL & $\mathrm{NL}$ & D $24^{\circ}$ & $\mathrm{NL}$ & $\mathrm{NL}$ & D $20^{\circ}$ \\
\hline$\left(30^{\circ}\right)$ & & & & & & & & $\mathrm{E} 20^{\circ}$ & & & E $15^{\circ}$ \\
\hline Joelho & D $50^{\circ}$ & D $42^{\circ}$ & D $90^{\circ}$ & $\mathrm{D} 88^{\circ}$ & D $26^{\circ}$ & D $10^{\circ}$ & D $96^{\circ}$ & D $60^{\circ}$ & D $47^{\circ}$ & D $90^{\circ}$ & D $90^{\circ}$ \\
\hline \multirow[t]{3}{*}{$\left(\text { ext-flex/ } 0^{\circ} 140\right)^{\circ}$} & $140^{\circ}$ & $140^{\circ}$ & $140^{\circ}$ & $140^{\circ}$ & $140^{\circ}$ & $125^{\circ}$ & $140^{\circ}$ & $140^{\circ}$ & $110^{\circ}$ & $140^{\circ}$ & $104^{\circ}$ \\
\hline & $\mathrm{E} 30^{\circ}$ & $\mathrm{E} 40^{\circ}$ & $\mathrm{E} 90^{\circ}$ & $\mathrm{E} 98^{\circ}$ & E $14^{\circ}$ & $\mathrm{E} 0^{\circ}$ & $\mathrm{E} 88^{\circ}$ & $\mathrm{E} 58^{\circ}$ & $\mathrm{E} 42^{\circ}$ & $\mathrm{E} 100^{\circ}$ & $\mathrm{E} 92^{\circ}$ \\
\hline & $140^{\circ}$ & $140^{\circ}$ & $140^{\circ}$ & $140^{\circ}$ & $140^{\circ}$ & $125^{\circ}$ & $140^{\circ}$ & $140^{\circ}$ & $105^{\circ}$ & $140^{\circ}$ & $104^{\circ}$ \\
\hline \multirow[t]{2}{*}{ Flexo de joelho } & D $50^{\circ}$ & D $42^{\circ}$ & $\mathrm{D} 90^{\circ}$ & $\mathrm{D} 88^{\circ}$ & D $26^{\circ}$ & D $10^{\circ}$ & D $96^{\circ}$ & D $60^{\circ}$ & D $47^{\circ}$ & $\mathrm{D} 90^{\circ}$ & D $90^{\circ}$ \\
\hline & $\mathrm{E} 30^{\circ}$ & $\mathrm{E} 40^{\circ}$ & $\mathrm{E} 90^{\circ}$ & $\mathrm{E} 98^{\circ}$ & $\mathrm{E} 14^{\circ}$ & E NL & $E 88^{\circ}$ & $\mathrm{E} 58^{\circ}$ & $\mathrm{E} 42^{\circ}$ & $\mathrm{E} 100^{\circ}$ & $\mathrm{E} 92^{\circ}$ \\
\hline Tornozelo & Eqüino & Eqüino & Eqüino & Eqüino & & Eqüino & Eqüino & Eqüino & Eqüino & Eqüino & Eqüino \\
\hline (flex-ext/ $20^{\circ} 0^{\circ}$ & D $27^{\circ}$ & $\mathrm{D}=\mathrm{E}$ & $\mathrm{D}=\mathrm{E}$ & $\mathrm{D}=\mathrm{E}$ & NL & D $35^{\circ}$ & D $35^{\circ}$ & D $40^{\circ}$ & D $35^{\circ}$ & D $25^{\circ}$ & $\mathrm{D} 33^{\circ}$ \\
\hline $\left.45^{\circ}\right)$ & E $26^{\circ}$ & $30^{\circ}$ & $35^{\circ}$ & $28^{\circ}$ & & E NL & $\mathrm{E} 33^{\circ}$ & $\mathrm{E} 50^{\circ}$ & $\mathrm{E} 40^{\circ}$ & $\mathrm{E} 30^{\circ}$ & $\mathrm{E} 40^{\circ}$ \\
\hline Pé (eversão- & Varo & Varo & Varo & Varo & & Varo & Varo & Varo & Varo & Varo & Varo \\
\hline inversão/ $20^{\circ} 0^{\circ}$ & fixo & fixo & fixo & D $45^{\circ}$ & $\mathrm{NL}$ & fixo & fixo & fixo & fixo & fixo & fixo \\
\hline \multirow[t]{2}{*}{$\left.40^{\circ}\right)$} & D $76^{\circ}$ & D $50^{\circ}$ & $\mathrm{D} 88^{\circ}$ & Evert & & $\mathrm{D} 30^{\circ}$ & D $14^{\circ}$ & $\mathrm{D} 38^{\circ}$ & $\mathrm{D}=\mathrm{E}$ & $\mathrm{D}=\mathrm{E}$ & $\mathrm{D}=\mathrm{E}$ \\
\hline & $\mathrm{E} 54^{\circ}$ & $\mathrm{E} 40^{\circ}$ & $\mathrm{E} 90^{\circ}$ & $\mathrm{E} 22^{\circ}$ & & E NL & E $24^{\circ}$ & E $23^{\circ}$ & $40^{\circ}$ & $25^{\circ}$ & $60^{\circ}$ \\
\hline
\end{tabular}

Cervic, cervical; D, direito; E, esquerdo; ext, extensão; flex, flexão; abd, abdução; ad, adução; Evert, evertido; NL, normal; rot, rotação.

Avaliação goniométrica e deformidades músculo-esqueléticas - Em todas as crianças encontramos retrações musculares nos quadris (pelo teste de Thomas), joelhos e cotovelos (ou seja, presença de limitação para extensão articular) (Tabela 3) (Fig 2). Havia limitação para elevação do ombro em cinco crianças e para adução em uma. Quanto aos movimentos cervicais, sete crianças apresentavam limitação para flexão, uma para extensão e seis para rotação lateral. Quatro crianças tinham limitação para flexão de punho e duas uma extensão. Observamos também pés eqüino-varo em dez crianças, sendo que, no caso 6 a deformidade era somente à direita, e no caso 4 o pé esquerdo encontrava- 
Tabela 4. Habilidades funcionais motoras máximas em onze crianças com distrofia muscular congênita com deficiência da merosina.

\begin{tabular}{|c|c|c|c|c|c|c|c|c|c|c|c|}
\hline $\begin{array}{l}\text { Habilid } \\
\text { máxima }\end{array}$ & 1 & 2 & 3 & 4 & 5 & 6 & 7 & 8 & 9 & 10 & 11 \\
\hline MS & $\begin{array}{c}\text { Flete } \\
\text { antebr } \\
\text { bilat }\end{array}$ & $\begin{array}{c}\text { Flete } \\
\text { antebr } \\
\text { bilat }\end{array}$ & $\begin{array}{l}\text { Flete } \\
\text { antebr } \\
\text { bilat }\end{array}$ & $\begin{array}{c}\text { Flete } \\
\text { antebr } \\
\text { bilat }\end{array}$ & $\begin{array}{l}\text { Flete } \\
\text { antebr } \\
\text { bilat }\end{array}$ & $\begin{array}{c}\text { Flete } \\
\text { antebr } \\
\text { bilat }\end{array}$ & $\begin{array}{l}\text { Flete } \\
\text { antebr } \\
\text { bilat }\end{array}$ & $\begin{array}{c}\text { Flete } \\
\text { antebr } \\
\text { bilat }\end{array}$ & $\begin{array}{c}\text { Flete } \\
\text { antebr } \\
\text { bilat }\end{array}$ & $\begin{array}{l}\text { Flete } \\
\text { antebr } \\
\text { bilat }\end{array}$ & $\begin{array}{c}\text { Flete } \\
\text { antebr } \\
\text { bilat }\end{array}$ \\
\hline Sentar & $\begin{array}{c}\text { Senta } \\
-\end{array}$ & $\begin{array}{l}\text { Senta } \\
\text { com } \\
\text { suport }\end{array}$ & $\begin{array}{l}\text { Senta } \\
\text { sem } \\
\text { suport }\end{array}$ & $\begin{array}{l}\text { Senta } \\
\text { com } \\
\text { suport }\end{array}$ & $\begin{array}{l}\text { Senta } \\
\text { sem } \\
\text { suport }\end{array}$ & $\begin{array}{l}\text { Senta } \\
\text { com } \\
\text { suport }\end{array}$ & $\begin{array}{l}\text { Senta } \\
\text { sem } \\
\text { suport }\end{array}$ & $\begin{array}{l}\text { Senta } \\
\text { sem } \\
\text { suport }\end{array}$ & $\begin{array}{c}\text { Senta } \\
\text { sem } \\
\text { suport }\end{array}$ & $\begin{array}{l}\text { Senta } \\
\text { sem } \\
\text { suport }\end{array}$ & $\begin{array}{l}\text { Senta } \\
\text { com } \\
\text { suport }\end{array}$ \\
\hline MI & - & - & - & - & $\begin{array}{l}\text { Posic } \\
\text { para } \\
\text { engat }\end{array}$ & $\begin{array}{l}\text { Fica de } \\
\text { pé com } \\
\text { órtese }\end{array}$ & - & $\begin{array}{c}\text { Arrasta } \\
\text { nádeg }\end{array}$ & $\begin{array}{c}\text { Arrasta } \\
\text { nádeg }\end{array}$ & - & - \\
\hline Cervical & - & - & $\begin{array}{l}\text { Sust a } \\
\text { cabeça } \\
\text { sentad }\end{array}$ & $\begin{array}{l}\text { Sust a } \\
\text { cabeça } \\
\text { sentad }\end{array}$ & $\begin{array}{l}\text { Sust a } \\
\text { cabeça } \\
\text { sentad }\end{array}$ & $\begin{array}{l}\text { Sust a } \\
\text { cabeça } \\
\text { sentad }\end{array}$ & $\begin{array}{l}\text { Sust a } \\
\text { cabeça } \\
\text { sentad }\end{array}$ & $\begin{array}{l}\text { Sust a } \\
\text { cabeça } \\
\text { sentad }\end{array}$ & $\begin{array}{l}\text { Sust a } \\
\text { cabeça } \\
\text { sentad }\end{array}$ & $\begin{array}{l}\text { Sust a } \\
\text { cabeça } \\
\text { sentad }\end{array}$ & $\begin{array}{l}\text { Sust a } \\
\text { cabeça } \\
\text { sentad }\end{array}$ \\
\hline
\end{tabular}

Antebr, antebraço; bilat, bilateralmente; nádeg, nádegas; habilid, habilidade; MS, membros superiores; MI, membros inferiores; posic, posiciona; sentad, sentada; sust, sustenta.

Tabela 5. Avaliação das atividades de vida diária (AVDs) pelo Indicador de Barthel (IB) em onze crianças com distrofia muscular congênita com deficiência da merosina.

\begin{tabular}{|c|c|c|c|c|c|c|c|c|c|c|c|}
\hline AVDs & 1 & 2 & 3 & 4 & 5 & 6 & 7 & 8 & 9 & 10 & 11 \\
\hline Alimentação & 5 & 5 & 5 & 10 & 10 & 10 & 5 & 5 & 5 & 5 & 5 \\
\hline \multicolumn{12}{|l|}{ Mover-se na $C R$} \\
\hline para cama e retornar & 0 & 0 & 0 & 0 & 0 & 0 & 5 & 0 & 0 & 0 & 0 \\
\hline Higiene pessoal & 0 & 0 & 0 & 0 & 0 & 5 & 0 & 0 & 0 & 0 & 0 \\
\hline \multicolumn{12}{|l|}{ Entrar e sair do } \\
\hline banheiro & 0 & 0 & 5 & 0 & 5 & 0 & 5 & 0 & 5 & 0 & 0 \\
\hline Banhar-se & 0 & 0 & 0 & 0 & 0 & 0 & 0 & 0 & 0 & 0 & 0 \\
\hline \multicolumn{12}{|l|}{ Andar sobre } \\
\hline superfícies niveladas & 0 & 0 & 0 & 0 & 0 & 0 & 0 & 0 & 0 & 0 & 0 \\
\hline Propulsão da CR & 0 & 0 & 0 & 0 & 0 & 0 & 0 & 0 & 0 & 0 & 0 \\
\hline \multicolumn{12}{|l|}{ Subir e descer } \\
\hline escadas & 0 & 0 & 0 & 0 & 0 & 0 & 0 & 0 & 0 & 0 & 0 \\
\hline Vestir-se e despir-se & 0 & 0 & 5 & 5 & 5 & 5 & 5 & 0 & 0 & 0 & 0 \\
\hline \multicolumn{12}{|l|}{ Continência } \\
\hline intestinal/vesical & $5 / 5$ & $10 / 10$ & $5 / 5$ & $10 / 10$ & $5 / 10$ & $10 / 10$ & $5 / 5$ & $5 / 5$ & $5 / 5$ & $5 / 10$ & $10 / 10$ \\
\hline Total (IB) & 15 & 25 & 25 & 35 & 35 & 40 & 35 & 15 & 20 & 20 & 25 \\
\hline
\end{tabular}

$\mathrm{CR}$, cadeira de rodas; 0 , totalmente dependente; 5 , parcialmente dependente; 10 , independente

se em evertido. O grau da deformidade dos pés está apresentado na Tabela 3. Havia desvio ulnar do punho em oito crianças (casos 2, 3, 4, 7, 8, 9, 10 e 11).

Capacidade funcional motora - Como demonstrado na Tabela 4, nenhuma criança conseguia tirar a cabeça da maca quando deitada. Apenas duas crianças não conseguiam sustentar a cabeça na posição sentada. Somente uma criança não conseguia sentar nem com apoio (caso 1), quatro necessitavam de suporte para permanecer na posição sentada e três conseguiam sentar sem apoio. Nenhuma crian- ça possuía a habilidade motora necessária para engatinhar, ficar em pé ou andar. Duas conseguiam arrastar-se utilizando as nádegas, e somente uma criança se posicionava para engatinhar (caso 5) e outra ficava em pé com apoio ou órteses (caso 6). Nenhuma criança conseguia elevar os braços acima da cabeça ou dos ombros, mas todas tinham a capacidade para flexão dos antebraços. Aquelas crianças que não possuíam a capacidade para extensão ou flexão dos punhos (casos 2, 8, 9, 10 e 11) apresentavam deformidades fixas na articulação, limitando a interpretação do exame. 
Atividades da vida diária - O IB variou de 15 a 40 pontos (Tabela 5), sendo nossos casos classificados como dependentes ou semidependentes para a maioria das AVDs estudadas. Somente três crianças conseguiam se alimentar de forma independente, as outras necessitavam de ajuda de terceiros. Seis crianças apresentavam controle esfincteriano. Destas, somente os casos 5 e 10 tinham controle vesical. A maioria das crianças conseguia vestir-se, despir-se e banhar-se com ajuda de terceiros, mas outras duas (casos 1 e 2) eram totalmente dependentes para vestuário e higiene. Todas as crianças não tinham a capacidade para a marcha, e eram incapazes de tocar sozinhas a cadeira de rodas.

Informações adicionais - Escoliose foi observada em sete crianças (casos 1, 2, 3, 4, 7, 10 e 11). Quatro delas (casos 1, 2, 4 e 11) apresentavam curva acentuada e não redutível, e duas (casos 2 e 4) tinham espinha rígida cervical associada. Obtivemos raio$X$ de coluna vertebral somente em três crianças (casos 3,4 e 10), as quais tinham curvas torácicas de $10^{\circ}$, $78^{\circ}$, e $30^{\circ}$, respectivamente.

A maioria das crianças apresentava queixa de broncopneumonia de repetição. Em cinco crianças, que tinham prova de função pulmonar, as capacidades vitais foram de $72 \%$ (caso 3 ), $25 \%$ (caso 4), $50 \%$ (caso 9), 40\% (caso10) e 35\% (caso 11), indicando síndrome restritiva pulmonar intensa nos casos 4, 10 e 11, moderada para o caso 9 e leve para o caso 3 .

O nível cognitivo foi considerado normal em todos os casos.

\section{DISCUSSÃO}

Nossos resultados confirmaram a gravidade do comprometimento motor na DMC-DM. São crianças com intenso e difuso comprometimento muscular, notado antes dos seis meses de vida, e com grave atraso nas aquisições motoras ${ }^{1,6,20}$. Na literatura, os estudos que apresentaram dados quanto à evolução destas crianças, revelaram que, apesar da gravidade motora, o padrão de força muscular é relativamente estável, ou com mínima progressão ${ }^{1,5,6,8}$. No entanto, as perdas funcionais observadas durante a vida da criança estão mais relacionadas com a ocorrência de deformidades músculo-esqueléticas, especialmente de retrações musculares, e também do comprometimento respiratório ${ }^{1,6,11}$. Tal descrição deixa clara a importância de uma abordagem ampla e precoce no sentido de evitar complicações decorrentes da intensa fraqueza muscular, tais como atrofia muscular por desuso, deformidades músculo-esqueléticas e redução da performance cardiovascular e pulmonar ${ }^{11,12}$.
Para que um programa de reabilitação tenha sucesso, especialmente com um grupo de pacientes portadores de uma doença tão incapacitante, é fundamental o conhecimento detalhado das principais limitações funcionais e dos grupos musculares mais acometidos. Desta forma nos propusemos a realizar tal estudo num grupo relativamente grande de crianças com DMC-DM que estão em acompanhamento médico regular na nossa instituição.

Os nossos dados mostraram que na DMC-DM o envolvimento da musculatura esquelética é difuso, incluindo-se a musculatura cervical, paravertebral e dos membros, neste caso afetando mais as porções proximais que as distais. Observamos que os grupos musculares de membros inferiores foram tão afetados quanto os de membros superiores. No nosso estudo, notamos que os grupos musculares extensores estavam mais acometidos que os flexores, isto certamente é um fator agravante na ocorrência de retrações musculares devido ao desequilíbrio entre grupos musculares agonistas e antagonistas. No entanto, não é possível, neste momento, determinar se a retração muscular dos flexores é causada pela maior fraqueza muscular dos extensores, ou se é a retração muscular dos flexores que primariamente ocasiona maior paresia nos músculos extensores. O seguimento a longo prazo de crianças com esta situação poderá indicar qual destas duas situações é o fator fundamental.

A presença de força muscular grau 2 pela MRC, como observado nos músculos proximais dos membros, já denota grave ausência de movimento contra a gravidade. Se estes músculos não forem exercitados adequadamente, certamente ocorrerão atrofia muscular (desuso) e deformidades. É importante lembrar que, apesar de muito usada, a escala MRC apresenta como limitação ser subjetiva e requerer experiência do examinador para quantificar e discriminar o grau de força muscular (especialmente entre os graus 4 e 5). Esta avaliação fica muito comprometida naquelas articulações com deformidades fixas, como pudemos constatar no nosso estudo. A utilização de equipamentos para medir a força isocinética e isométrica, apesar de ter maior sensibilidade, também apresenta sérias limitações tais como: o preço do equipamento, a necessidade da colaboração efetiva da criança, de força muscular adequada e de ausência de deformidades ${ }^{17}$.

As retrações musculares mais graves e freqüentes observadas nos nossos pacientes ocorreram nos membros inferiores. Em geral, notou-se uma pos- 
tura em flexão dos quadris e joelhos e eqüinovaro dos pés. Essas deformidades são também decorrentes de uma postura inadequada na posição sentada. Nestas crianças tais retrações ocorreram em grandes articulações responsáveis por boa parte das habilidades funcionais em membros inferiores (engatinhar, ficar de pé e andar). Uma característica marcante destas crianças foi exatamente a ausência da habilidade para ficar de pé ou andar.

Nos membros superiores a articulação mais comprometida foi o cotovelo. A ocorrência desta limitação na amplitude do movimento do cotovelo, associada ao comprometimento da musculatura proximal de ombro e deformidades nas mãos e punhos produz limitações acentuadas das atividades de vida diária que poderiam ser realizadas pela própria criança de forma independente ${ }^{17}$.

Curiosamente, limitações nos movimentos dos ombros, quando avaliados passivamente, foram menos freqüentes, talvez porque apesar da gravidade da fraqueza muscular, não havia desequilíbrio entre grupos musculares agonistas e antagonistas. Outras deformidades em membros superiores encontradas no nosso estudo foram desvio ulnar e postura fletida dos punhos. Apesar de não ter sido realizado análise goniométrica das articulações dos dedos, não notamos nestas crianças a presença de mãos em garra ou retrações significativas das falanges.

O envolvimento da musculatura cervical é outro fator altamente incapacitante para em crianças com DMC ${ }^{6,11}$. Todas as nossas crianças tiveram força grau 2 tanto para flexão quanto para extensão do pescoço, indicando incapacidade para elevação do pescoço quando na posição supina (vencer a gravidade), embora algumas delas conseguissem manter o sustento cervical quando sentadas. Observamos a ocorrência freqüente de limitação dos movimentos cervicais especialmente para a flexão (decorrente de retração dos músculos extensores) e para rotação (devido à retração dos esternocleidomastoideos). Este é sabidamente um achado característico de crianças com DMC, não sendo normalmente encontrado em outras formas de miopatias congênitas. A distrofia muscular de Emery-Dreifuss e a DMC com a forma de espínha rígida; no entanto, podem cursar com graves retrações na musculatura cervi$\mathrm{cal}^{3,20}$. A limitação para os movimentos cervicais observada na maioria dos nossos casos é um fator altamente incapacitante nestas crianças, pois dificulta o sustento cervical, e atividades como alimentação e deglutição. Força grau 2 também foi notada na musculatura lombar e abdominal, justificando a limitação destas crianças para a elevação do tórax e do dorso quando na posição supina (vencer a gravidade) e para realizarem funções como sentar e rolar.

Fator altamente limitante na DMC é a escoliose, notada em sete dos nossos casos. A escoliose é uma das principais complicações ortopédicas da DMC, e ocorre devido ao comprometimento dos músculos paravertebrais, surgindo, em geral, nos primeiros anos de vida, com piora no período pré-puberal| ${ }^{1,6,11,12}$. É uma situação de difícil tratamento, pois em geral está associada à redução da capacidade vital respiratória, o qual limita o procedimento cirúrgico. No nosso estudo, nas crianças que tinham raio- $X$ de coluna vertebral, a curva variou de $10^{\circ}$ a $72^{\circ}$ de deformidade. Nenhuma delas havia sido submetida a tratamento cirúrgico até o presente momento.

Nossas crianças apresentavam intenso comprometimento funcional motor tanto de membros inferiores quanto de membros superiores. Com relação aos membros inferiores, nenhum dos nossos casos adquiriu marcha, e apenas uma permanecia de pé com órteses ou apoio. O ortostatismo fica muito prejudicado nestes casos devido às retrações nos quadris e joelhos, e deformidades dos pés. Para os membros superiores a maior limitação funcional foi à elevação lateral dos braços acima dos ombros, o que certamente dificulta importantes AVDs tais como elevação das mãos até a boca para alimentação e higiene oral, e pentear os cabelos, como pudemos observar na maioria dos nossos casos. Pelo IB pudemos observar nas nossas crianças um alto índice de dependência nas AVDs. O IB não nos pareceu ser um bom teste de AVDs para estas crianças, pois utiliza alguns "scores" como controle esfincteriano e fecal que em geral não são afetados nestes pacientes. Assim, se eliminássemos os "scores" para controle esfincteriano, o IB seria extremamente reduzido, denotando a intensa dependência destas crianças para as AVDs mais fundamentais, tais como alimentação e higiene.

Os principais fatores prognósticos na DMC são a insuficiência respiratória e a escoliose ${ }^{6,12}$. Infelizmente não tivemos a oportunidade de avaliar a função respiratória em todas as crianças deste estudo, ou pela própria idade, ou pela limitação para obtenção do exame. Nos quatro casos em que pudemos avaliar a função respiratória, todos tinham padrão restritivo. Apesar da doença não ter caráter progressivo, o envolvimento respiratório é realmente incapacitante, e como descrito por outros auto- 
res, tende a piorar durante a vida, especialmente na adolescência ${ }^{1,6,11,12}$. Não há na literatura estudos conclusivos sobre a sobrevida destas crianças, porém em geral o óbito ocorre após a adolescência por insuficiência respiratória ${ }^{1,6}$.

Nosso estudo conclui que o comprometimento motor na DMC com deficiência da merosina é grave, com grande incapacidade para marcha, mas também para o uso de membros superiores. As deformidades são freqüentes e ocorrem precocemente, agravando ainda mais as limitações funcionais decorrentes da fraqueza muscular. Assim, um programa amplo de reabilitação deve ser iniciado precocemente, com abordagem multi-profissional, dando atenção especial aos grupos musculares mais afetados, as habilidades motoras mais comprometidas e a função pulmonar.

\section{REFERÊNCIAS}

1. Tomé FMS, Guicheney P, Fardeau M. Congenital muscular dystrophies. In Emery AEH (ed). Neuromuscular disorders: clinical and molecular genetics. Chichester: John Wiley \& Sons, 1998:21-58.

2. Fukuyama Y. Congenital muscular dystrophies: an update. J Child Neurol 1999;14:28-30

3. Mercuri E, Sewry C, Brown SC, Muntoni F. Congenital muscular dystrophies. Semin Pediatr Neurol 2002;9:120-131.

4. Muntoni F, Valero de Bernabe B, Bittner R, et al. 114th ENMC International Workshop on Congenital Muscular Dystrophy (CMD) 17-19 January 2003, Naarden, The Netherlands: (8th Workshop of the International Consortium on CMD; 3rd Workshop of the MYO-CLUSTER project GENRE). Neuromuscul Disord 2003; 13:579-588.

5. Philpot J, Sewry C, Pennock J, Dubowitz V. Clinical phenotype in con- genital muscular dystrophy: correlation with expression of merosin in skeletal muscle. Neuromusc Disord 1995;5:301-305.

6. Fardeau M, Tome FM, Helbling-Leclerc A, et al. Congenital muscular dystrophy with merosin deficiency: clinical, histopathological, immunocytochemical and genetic analysis. Rev Neurol (Paris) 1996;152:11-19.

7. Reed UC, Marie SK, Vainzof M, et al. Congenital muscular dystrophy with cerebral white matter hypodensity: correlation of clinical features and merosin deficiency. Brain Dev 1996;18:53-58.

8. Talim B, Kale G, Topaloglu H, et al. Clinical and histopathological study of merosin-deficient and merosin-positive congenital muscular dystrophy. Pediatr Dev Pathol 2000;3:168-176.

9. Jones KJ, Morgan $\mathrm{G}$, Johnston $\mathrm{H}$, et al. The expanding phenotype of laminin alpha2 chain (merosin) abnormalities: case series and review. J Med Genet 2001;38:649-657.

10. Caro PA, Scavina M, Hoffman E, Pegoraro E, Marks HG. MR imaging findings in children with merosin-deficient congenital muscular dystrophy. Am J Neuroradiol 1999;20:324-326.

11. Jones R, Khan R, Hughes S, Dubowitz V. Congenital muscular dystrophy: the importance of early diagnosis and orthopaedic management in the long-term prognosis. J Bone Joint Surg Br 1979;61:13-17.

12. Eagle M. Report on the muscular dystrophy campaign workshop: exercise in neuromuscular diseases Newcastle, January 2002. Neuromusc Disord 2002;12:975-983.

13. Fowler WM, Abresch RT, Aitkens S, et al. Profiles of neuromuscular diseases: design of a protocol. Am J Phys Med Rehabil 1995;74:62-69.

14. Medical Research Council: Aids to the investigation of peripheral nerve injuries. War Memorandum (revised 2nd edition). London: HMSO, 1943.

15. Scott OM, Goddard C, Dubowitz V. Quantitation of muscle function in children: a prospective study in Duchenne muscular dystrophy. Muscle Nerve 1982;5:291-301

16. American Academy of Orthopaedic Surgeons. The clinical measurement of joint motion. Chicago: American Association of Orthopaedic Surgery, 1994.

17. Barros TEP Filho, Lech O. Exame físico em ortopedia. São Paulo: Sarvier, 2002.

18. Russman BS, Buncher CR, White M, Samaha FJ, Iannaccone ST. Function changes in spinal muscular atrophy II and III. The DCN/SMA Group. Neurology 1996;47:973-976.

19. Mahoney FI, Barthel DW. Functional evaluation: Barthel index. Md State Med J 1965;14:61-65.

20. Voit T. Congenital muscular dystrophies: 1997 update. Brain Dev 1998; 20:65-74 